\title{
Witten-Veneziano mechanism and pseudoscalar glueball-meson mixing in holographic QCD
}

\author{
Josef Leutgeb $\odot$ and Anton Rebhan $(1)$ \\ Institut für Theoretische Physik, Technische Universität Wien, Wiedner Hauptstr. 8-10,
} A-1040 Vienna, Austria

(Received 4 November 2019; published 10 January 2020)

\begin{abstract}
We revisit the $\mathrm{U}(1)_{A}$ anomaly in the holographic model of low-energy QCD by Witten, Sakai, and Sugimoto, presenting a new and direct derivation of the Witten-Veneziano mechanism for generating the mass of the $\eta^{\prime}$ through an anomalous mixing of the Ramond-Ramond $C_{1}$ field with the singlet component of the pseudoscalar mesons. The latter turns out to have a kinetic mixing with the normalizable modes of the $C_{1}$ field representing pseudoscalar glueballs, yielding additional vertices for their production and their decay that dominate over those of the unmixed case considered previously in the Witten-Sakai-Sugimoto model. The leading channel is predicted to be a decay into two vector mesons, followed in importance by decay into three pseudoscalar mesons. The issue of production of pseudoscalar glueballs in radiative $J / \psi$ decays and in double diffractive processes is also discussed briefly.
\end{abstract}

DOI: 10.1103/PhysRevD.101.014006

\section{INTRODUCTION}

In $\mathrm{QCD}$, the $\mathrm{U}(1)_{A}$ part of the tree-level flavor symmetry $\mathrm{U}\left(N_{f}\right)_{L} \times \mathrm{U}\left(N_{f}\right)_{R}$ is broken by the axial anomaly, leading to only $N_{f}^{2}-1$ pseudoscalar Goldstone bosons in the spontaneous breaking of $\mathrm{U}(1)_{V} \times \mathrm{SU}\left(N_{f}\right)_{L} \times \mathrm{SU}\left(N_{f}\right)_{R} \rightarrow$ $\mathrm{U}\left(N_{f}\right)_{V}$ with masses determined by the Gell-Mann-OakesRenner relation, while one isoscalar pseudoscalar boson, the $\eta^{\prime}$, is found to be too heavy to be a Goldstone boson [1]. Its mass is determined by nonperturbative effects involving the axial anomaly, and it was suggested by 't Hooft [2,3] that these are due to instantons. However, in the limit of large number of colors $N_{c}$ the effects of a dilute gas of instantons is exponentially suppressed. A different mechanism was proposed by Veneziano [4] and Witten [5], who showed that the nontrivial $\theta$ dependence of large- $N_{c}$ pure Yang-Mills theory implies a mass term for the singlet $\eta_{0}$ according to $m_{0}^{2}=2 N_{f} \chi_{g} / f_{\pi}^{2}$, where $\chi_{g}$ is the topological susceptibility of pure Yang-Mills theory.

In $[6,7]$ it was shown that this mechanism is indeed realized in the AdS/CFT framework. In the Witten model [8] of low-energy QCD, which is based on a supersymmetrybreaking circle compactification of $N_{c}$ D4-branes in typeIIA supergravity, this was initially discussed in a model with flavor D6-branes [7] and subsequently also in the chiral

Published by the American Physical Society under the terms of the Creative Commons Attribution 4.0 International license. Further distribution of this work must maintain attribution to the author(s) and the published article's title, journal citation, and DOI. Funded by SCOAP.
Witten-Sakai-Sugimoto (WSS) model [9,10], which is based on D8-branes and which realizes a fully non-Abelian chiral symmetry breaking.

A more detailed discussion of the Witten-Veneziano mechanism in the WSS model was recently given by Bartolini et al. in [11]. In the present paper we present an alternative, more direct derivation, which moreover allows us to reanalyze the possible mixing of the singlet $\eta_{0}$ with the pseudoscalar glueball $\tilde{G}$. In the bottom-up V-QCD model of Ref. [12], where the Veneziano limit of $N_{c} \rightarrow \infty$ with $N_{f} / N_{c}$ fixed is employed, such mixing in the context of the Witten-Veneziano relation has been discussed in [13]. In the WSS model, one of us with F. Brünner has found [14] a vanishing mass mixing of $\eta_{0}$ and $\tilde{G}$ which led to the conclusion of a very narrow pseudoscalar state, because to leading order in the WSS model only vertices involving jointly the scalar glueball and $\eta_{0}$ appeared to be present. Our new derivation reveals the presence of a kinetic (derivative) mixing which leads to additional decay modes of pseudoscalar glueballs that are dominating those considered in Ref. [14] or in the phenomenological model of Ref. [15].

The importance of gluonic contributions for the physics of $\eta^{\prime}$ mesons is already evidenced by the Witten-Veneziano mechanism. It has also been emphasized by the analysis of Ref. [16], who have however left open the question whether this involves a coupling to physical pseudoscalar glueball excitations (see also [17-19]). Possible pseudoscalar glueball-meson mixing scenarios have been discussed widely in the literature [20-27], but exclusively in the form of mass mixings. The mixing scenario that we obtain in the WSS model leads to a dominance of the couplings 
that are induced by the Chern-Simons term, in particular to two vector mesons. Inclusion of quark masses leads to additional couplings such as to three pseudoscalar mesons.

Assuming that the couplings obtained in the WSS model are more reliable than the results for the glueball masses, which for tensor and pseudoscalar glueballs are much lower than those predicted by lattice QCD, we consider a range of pseudoscalar glueball masses, with the result that a pseudoscalar glueball of around $2.6 \mathrm{GeV}$ as indicated by (quenched) lattice QCD [28,29] appears to be a rather broad resonance instead of a narrow state, which may hinder its experimental identification. On the other hand, its relatively strong coupling to vector mesons should enhance its production cross section in central exclusive production as well as in radiative $J / \psi$ decays. The decay patterns derived in this paper might help to identify a pseudoscalar glueball in experimental studies.

So far, the experimental search for glueballs in the hadron spectrum has led to two candidates for the lightest scalar glueball, $f_{0}(1500)$ and $f_{0}(1710)$. Different phenomenological models are split on the question of which of the two would have the largest glueball component, and the WSS results for glueball decay patterns of Refs. [30,31] favor $f_{0}(1710)$. For the tensor glueball there are several candidates above $1900 \mathrm{MeV}$, with the WSS model indicating [32] that it should be a relatively broad resonance, if the mass is around $2.4 \mathrm{GeV}$ as indicated by both quenched and unquenched lattice QCD [28,29,33-35]. Prior to the first lattice QCD studies, where the pseudoscalar glueball has been found to be heavier than the tensor glueball, the pseudoscalar meson $\imath$ (1440), now interpreted as two states $\eta(1405)$ and $\eta(1475)$ (see however [36]), was regarded as a strong glueball candidate; in fact, the $\eta(1405)$ is still often considered as such [37]. In the Witten model, the pseudoscalar glueball is heavier than the tensor glueball. The kinetic mixing obtained by us when quarks are included increases the mass of the pseudoscalar glueball somewhat, while leaving unchanged (to leading order) the $\eta\left(^{\prime}\right)$ mesons, which suggests that the pseudoscalar glueball is still to be discovered with a mass that is above that of the tensor glueball.

In Sec. II we review the WSS model, in particular the role of the Ramond-Ramond field $C_{1}$ which determines the $\theta$ parameter of the dual theory, and how the $\mathrm{U}(1)_{A}$ anomaly and the Witten-Veneziano mechanism is realized. In Sec. III we derive the mixing of $\eta_{0}$ and the pseudoscalar glueball modes contained in the normalizable $C_{1}$ modes, and we work out its consequences for glueball-meson vertices and decay patterns of the pseudoscalar glueball in Sec. IV, followed by a discussion and an outlook in Sec. V.

\section{WITTEN-SAKAI-SUGIMOTO MODEL AND WITTEN-VENEZIANO MECHANISM}

\section{A. The Witten model of low-energy QCD}

The WSS model is based on the Witten model of lowenergy QCD [8] provided by the near-horizon geometry of a large number $\left(N_{c}\right)$ of coincident D4-branes in type-IIA superstring theory wrapped on a circle of circumference $R_{4}=2 \pi M_{\mathrm{KK}}^{-1}$ with antiperiodic boundary conditions for fermions. Since gauginos are massive at tree level and adjoint scalars acquire masses through loops, the dual theory at energies much smaller than $M_{\mathrm{KK}}$ is pure nonsupersymmetric Yang-Mills theory at large 't Hooft coupling $\lambda=N_{c} g_{\mathrm{YM}}^{2}$. In the supergravity approximation, where the Kaluza-Klein mass scale $M_{\mathrm{KK}}$ is kept finite, the background can be obtained from the dimensional reduction of an 11-dimensional doubly Wick-rotated black-hole geometry in $\mathrm{AdS}_{7} \times S^{4}$,

$$
\begin{aligned}
d s_{11}^{2}= & \frac{r^{2}}{L^{2}}\left[f(r) d x_{4}^{2}+\eta_{\mu \nu} d x^{\mu} d x^{\nu}+d x_{11}^{2}\right] \\
& +\frac{L^{2}}{r^{2}} \frac{d r^{2}}{f(r)}+\frac{L^{2}}{4} d \Omega_{4}^{2}, \\
f(r)= & 1-\frac{r_{\mathrm{KK}}^{6}}{r^{6}},
\end{aligned}
$$

where $\mu, \nu=0, \ldots, 3, \eta_{\mu \nu}=\operatorname{diag}(-1,1,1,1)$, together with a nonzero 4-form field strength $F_{4}=3(L / 2)^{3} \omega_{4}$, where $\omega_{4}$ is the volume form of a unit 4-sphere with volume $V_{4}=$ $8 \pi^{2} / 3$. A regular Euclidean black hole is produced by identifying $\tau \equiv x_{4} \simeq x_{4}+2 \pi R_{4}$ with $R_{4}=M_{\mathrm{KK}}^{-1}=L^{2} /\left(3 r_{\mathrm{KK}}\right)$.

Dimensional reduction from 11-dimensional supergravity [38] with $\kappa_{11}^{2}=\left(2 \pi l_{P}\right)^{9} /(4 \pi)$ and $l_{P}=g_{s}^{1 / 3} l_{s}$ through $x_{11} \simeq x_{11}+2 \pi R_{11}$, with $R_{11}=g_{s} l_{s}$, and

$$
\begin{aligned}
d s_{11}^{2}= & G_{\hat{M} \hat{N}} d x^{\hat{M}} d x^{\hat{N}}=e^{-2 \Phi / 3} g_{M N} d x^{M} d x^{N} \\
& +e^{4 \Phi / 3}\left(d x^{11}+A_{M} d x^{M}\right)^{2},
\end{aligned}
$$

where $e^{\Phi}=(r / L)^{3 / 2}$ and hatted (unhatted) indices refer to 11 (10) dimensions, leads to type-IIA supergravity with string-frame action

$$
S=S_{\mathrm{NS}}+S_{\mathrm{R}}+S_{\mathrm{CS}},
$$

where

$$
\begin{aligned}
& S_{\mathrm{NS}}= \frac{1}{2 \kappa_{10}^{2}} \int d^{10} x \sqrt{-g} e^{-2 \Phi} \\
& \times\left(R+4 \partial_{M} \Phi \partial^{M} \Phi-\frac{1}{2}\left|d B_{2}\right|^{2}\right), \\
& S_{\mathrm{R}}=-\frac{1}{4 \kappa_{10}^{2}} \int d^{10} x \sqrt{-g}\left(\left|F_{2}\right|^{2}+\left|F_{4}\right|^{2}\right), \\
&\left|F_{p}\right|^{2}=\frac{1}{p !} F_{M \ldots} F^{M \ldots}, \\
& S_{\mathrm{CS}}=-\frac{1}{4 \kappa_{10}^{2}} \int B_{2} \wedge F_{4} \wedge F_{4} .
\end{aligned}
$$

In order to have a standard form of D-brane actions with prefactors $\mu_{p}=(2 \pi)^{-p} l_{s}^{-(p+1)}$ for both the Dirac-Born-Infeld 
(DBI) and Chern-Simons (CS) parts, we absorb the factor $g_{s}^{2}$ originally contained in $2 \kappa_{10}^{2}=2 \kappa_{11}^{2} /\left(2 \pi R_{11}\right)$ by rescaling $F_{2,4}=g_{s} F_{2,4}^{10 \mathrm{D}}$ and $e^{-\Phi}=g_{s} e^{-\Phi_{10 \mathrm{D}}}$, upon which we drop the 10D labels on the fields and redefine $2 \kappa_{10}^{2}=(2 \pi)^{7} l_{s}^{8}$.

In the coordinates used in $[9,10]$,

$$
U=\frac{r^{2}}{2 L}, \quad K(Z) \equiv 1+Z^{2}=\frac{r^{6}}{r_{\mathrm{KK}}^{6}}=\frac{U^{3}}{U_{\mathrm{KK}}^{3}},
$$

the 10-dimensional metric reads

$$
\begin{aligned}
d s_{10}^{2}= & \left(\frac{U}{R_{\mathrm{D} 4}}\right)^{3 / 2}\left[\eta_{\mu \nu} d x^{\mu} d x^{\nu}+f(U) d x_{4}^{2}\right] \\
& +\left(\frac{R_{\mathrm{D} 4}}{U}\right)^{3 / 2}\left[\frac{d U^{2}}{f(U)}+U^{2} d \Omega_{4}^{2}\right]
\end{aligned}
$$

with $f(U)=1-\left(U_{\mathrm{KK}} / U\right)^{3}$ and $R_{\mathrm{D} 4} \equiv L / 2$; the dilaton and 4-form field strength are given by

$$
e^{\Phi}=g_{s}\left(U / R_{\mathrm{D} 4}\right)^{3 / 4}
$$

and

$$
F_{4}=d C_{3}=\frac{3 R_{\mathrm{D} 4}^{3}}{g_{s}} \omega_{4}
$$

[Here and in the following we stick to the usual normalization of the Ramond-Ramond fields in the string-theory literature [38]. In $[9,10]$ these fields are rescaled according to $C_{p}=(2 \pi)^{p-1} l_{s}^{p} C_{p}^{\mathrm{SS}}$.] The 4-form field strength is related to the number $N_{c}$ of D4-branes by

$$
(2 \pi)^{2} l_{s}^{3} \int_{S^{4}} F_{4}=2 \pi N_{c},
$$

which implies $R_{\mathrm{D} 4}^{3} \equiv(L / 2)^{3}=\pi g_{s} N_{c} l_{s}^{3}$.

The parameters ${ }^{1}$ of the dual boundary theory, which upon dimensional reduction through the circle $S_{\tau}$ with radius $M_{\mathrm{KK}}^{-1}$ becomes pure $3+1$-dimensional Yang-Mills theory

$$
\mathcal{L}=-\frac{1}{2 g_{\mathrm{YM}}^{2}} \operatorname{Tr}\left|F_{\mathrm{YM}}\right|^{2}+\frac{\theta}{8 \pi^{2}} \operatorname{Tr} F_{\mathrm{YM}} \wedge F_{\mathrm{YM}},
$$

can be identified from the UV limit $U \rightarrow \infty$ of the D4brane action

\footnotetext{
${ }^{\mathrm{I}}$ Following the notation of $[9,10,39], g_{\mathrm{YM}}^{2}$ differs by a factor two from the usual particle physics convention so that $\alpha_{s}=g_{\mathrm{YM}}^{2} /(2 \pi)$.
}

$$
\begin{aligned}
S^{\mathrm{D} 4}= & -\mu_{4} \operatorname{Tr} \int d^{4} x d \tau e^{-\Phi} \sqrt{-g_{(5)}} \\
& \times\left(\mathbf{1}+\frac{1}{2}\left(2 \pi \alpha^{\prime}\right)^{2}\left|F_{\mathrm{YM}}\right|^{2}+\ldots\right) \\
& +\mu_{4}\left(2 \pi \alpha^{\prime}\right)^{2} \int C_{1} \wedge F_{\mathrm{YM}} \wedge F_{\mathrm{YM}}+\ldots,
\end{aligned}
$$

with $\mu_{4}=(2 \pi)^{-4} l_{s}^{-5}$ and $\alpha^{\prime}=l_{s}^{2}$. This gives

$$
g_{\mathrm{YM}}^{2}=2 \pi g_{s} l_{s} M_{\mathrm{KK}}, \quad \theta+2 \pi k=\frac{1}{l_{s}} \int_{S_{\tau}} C_{1}
$$

with $k$ an integer.

The Witten background reviewed above has a vanishing one-form field $C_{\tau}$ and thus corresponds to the theory with vanishing $\theta$ parameter, or sufficiently small $\theta / N_{c}$ such that the backreaction on the background can be neglected, in the $k=0$ branch. (The fully backreacted case has been worked out in Ref. [40].)

The holographic dictionary thus relates nonnormalizable fluctuations of $C_{\tau}$ to a local, possibly $x$-dependent $\theta$ parameter. Normalizable modes are instead interpreted as pseudoscalar $\left(J^{P C}=0^{-+}\right)$glueball excitations [41].

The relevant quadratic action for these fields is

$$
\begin{aligned}
S_{\mathrm{R}} \supset & -\frac{1}{4 \kappa_{10}^{2}} \int d^{10} x \sqrt{-g}\left(\left|F_{2}\right|^{2}\right) \\
= & -\frac{2 \pi R_{4} V_{4}}{64 \kappa_{10}^{2}} \int d^{4} x \int_{r_{\mathrm{KK}}}^{\infty} d r \\
& \times\left(\frac{r^{3} L}{f(r)} \eta^{\mu \nu} \partial_{\mu} C_{\tau} \partial_{\nu} C_{\tau}+\frac{r^{7}}{L^{3}}\left(\partial_{r} C_{\tau}\right)^{2}\right) .
\end{aligned}
$$

The resulting field equations have the nonnormalizable solution

$$
C_{\tau}^{(0)}=\frac{l_{s}}{2 \pi R_{4}} f(r) \vartheta(x) \quad \text { for } \square \vartheta(x)=0 .
$$

In the Witten model of pure Yang-Mills theory, $\vartheta=\theta$, but since this connection will be modified when quarks are included, we have providently introduced a different symbol.

Inserting (14) in the action gives

$$
S_{\mathrm{R}}=-\frac{\chi_{g}}{2} \int d^{4} x \theta^{2}, \quad \chi_{g}=\frac{\lambda^{3} M_{\mathrm{KK}}^{4}}{4(3 \pi)^{6}},
$$

with $\chi_{g}$ being the topological susceptibility.

The normalizable solutions will be expanded in mass eigenfunctions, with radial eigenvalue equations

$$
\partial_{r}\left(\frac{r^{7}}{L^{3}} \partial_{r} C_{\tau}^{(2)}\right)+\frac{r^{3} L}{f(r)} M_{G}^{2} C_{\tau}^{(2)}=0
$$


subject to boundary conditions $C_{\tau}^{(2)}(r=\infty)=C_{\tau}^{(2)}(r=$ $\left.r_{\mathrm{KK}}\right)=0, \quad \partial_{r} C_{\tau}^{(2)}\left(r=r_{\mathrm{KK}}\right) \neq 0$. This determines the mass of the lightest pseudoscalar glueball as $M_{G}=$ $1.885 \ldots \times M_{\mathrm{KK}}$.

\section{B. Inclusion of quarks and $\mathrm{U}(1)_{A}$ anomaly}

Sakai and Sugimoto have extended the Witten model by introducing left- and right-handed chiral quarks through $N_{f}$ pairs of D8 and $\overline{\mathrm{D} 8}$ probe branes localized at separate points on the circle $S_{\tau}$ at the holographic boundary. Chiral symmetry breaking $\mathrm{U}\left(N_{f}\right)_{L} \times \mathrm{U}\left(N_{f}\right)_{R} \rightarrow \mathrm{U}\left(N_{f}\right)_{L+R}$ emerges from the fact that the D8- and $\overline{\mathrm{D} 8}$-branes have to join in the cigar-shaped background geometry. Choosing antipodal points on $S_{\tau}$ leads to embedding functions with constant $x_{4} \equiv \tau$ and a joining of the branes at the tip of the cigar at $r_{\mathrm{KK}}$ (or $U_{\mathrm{KK}}$ ). In this case one can extend the coordinate $Z$ introduced in (5) to the range $-\infty \ldots \infty$ in order to cover the radial extent of joined D8and $\overline{\mathrm{D} 8}$-branes.

The action for these flavor branes is given by $S^{\mathrm{D} 8}=$ $S_{\mathrm{DBI}}^{\mathrm{D} 8}+S_{\mathrm{CS}}^{\mathrm{D} 8}$ with

$S_{\mathrm{DBI}}^{\mathrm{D} 8}=-\mu_{8} \int_{\mathrm{D} 8} e^{-\Phi} \widetilde{\mathrm{Tr}} \sqrt{-\operatorname{det}\left(g_{(9)}+2 \pi \alpha^{\prime} \mathcal{F}+B_{2}\right)}$,

where $\widetilde{T r}$ denotes symmetrized trace, $\mu_{8}=(2 \pi)^{-8} l_{s}^{-(9)}$, and $\mathcal{F}=d \mathcal{A}+\mathcal{A} \wedge \mathcal{A}$ is the field strength tensor for the non-Abelian flavor gauge fields living on the D8-branes. $\left[B_{2}\right.$ is the bulk Kalb-Ramond 2-form field; its normalizable modes contain the pseudovector $\left(1^{+-}\right)$glueballs of the dual gauge theory [41,42].]

The even and odd radial mode functions of $\mathcal{A}_{\mu}$ are associated with the towers of vector and axial-vector meson fields, with the lowest mass eigenvalue $\left(m_{v_{1}}^{2} \approx 0.669 M_{\mathrm{KK}}^{2}\right)$ being identified as the $\rho$ meson mass such that $M_{\mathrm{KK}}=$ $949 \mathrm{MeV}$.

The massless pseudoscalar Goldstone bosons are described by

$U(x)=\mathrm{P} \exp i\left(\int_{-\infty}^{\infty} d Z A_{Z}(Z, x)\right)=\exp \left(i \Pi^{a} \lambda^{a} / f_{\pi}\right)$,

where $f_{\pi}^{2}=\frac{1}{54 \pi^{4}} \lambda N_{c} M_{\mathrm{KK}}^{2}$ and $\lambda^{a}$ are Gell-Mann matrices supplemented by $\lambda^{0}=\left(N_{f} / 2\right)^{-1 / 2} 1$. Setting $f_{\pi}=92.4 \mathrm{MeV}$ fixes $\lambda \approx 16.63$ for $N_{c}=3$. A smaller value of about 12.55 would be found by matching instead the large- $N_{c}$ lattice result [43] for the string tension. As in [32] we shall consider the range $\lambda=16.63 \ldots 12.55$ in order to obtain a theoretical error band for our quantitative predictions.

The 9-dimensional Chern-Simons term of the flavor brane is given by
$S_{\mathrm{CS}}^{\mathrm{D} 8}=\mu_{8} \sum_{q} \int_{\mathrm{D} 8} \sqrt{\hat{\mathcal{A}}(R)} \operatorname{Tr} \exp \left(2 \pi \alpha^{\prime} \mathcal{F}+B\right) \wedge C_{q}$,

where the so-called A-roof genus factor involves $\hat{A}(\mathcal{R})=$ $1+\frac{1}{192 \pi^{2}} \operatorname{Tr} \mathcal{R} \wedge \mathcal{R}+\ldots$ with $\mathcal{R}^{M N}=\frac{1}{2} R_{K L}{ }^{M N} d x^{K} d x^{L}$. The term involving $C_{3}$ contains the Wess-Zumino-Witten term of the dual gauge theory because $F_{4}=d C_{3}$ is nonzero in the background $[9,44]$.

The Chern-Simons term is also involved in the $\mathrm{U}(1)_{A}$ anomaly of the dual gauge theory, because the $C_{7}$ term modifies the equations of motion of the $C_{1}$ field which is responsible for the $\theta$ parameter. Using Hodge duality, $d C_{7}=F_{8}=\star F_{2}$, and integrating by parts, one finds that

$$
\begin{aligned}
S_{\mathrm{CS}} & \supset \mu_{8} 2 \pi \alpha^{\prime} \int_{\mathrm{D} 8} \operatorname{Tr}\left(\mathcal{F}_{2} \wedge C_{7}\right) \\
= & \mu_{8} 2 \pi \alpha^{\prime} \int \operatorname{Tr}\left(\mathcal{A}_{1}\right) \wedge \star F_{2} \wedge \omega_{\tau} \\
= & \mu_{8} 2 \pi \alpha^{\prime} \int d x^{10} \sqrt{\left|g_{10}\right|}[\delta(\tau)+\delta(\tau-\pi)] \\
& \times\left(\operatorname{Tr}\left(\mathcal{A}_{r}\right) g^{r r} g^{\tau \tau} \partial_{r} C_{\tau}+g^{\mu \nu} g^{\tau \tau} \operatorname{Tr}\left(\mathcal{A}_{\mu}\right) \partial_{\nu} C_{\tau}\right),
\end{aligned}
$$

where $\omega_{\tau}=[\delta(\tau)+\delta(\tau-\pi)] d \tau$ has been introduced to extend the integration to the entire bulk spacetime. Thus the flavor probe branes induce a mixing term of the Abelian part of the flavor gauge field $\hat{\mathcal{A}}:=N_{f}^{-1} \operatorname{Tr} \mathcal{A}$ living on the D8-brane with the field $C_{\tau}$. The linear equations of motion for $C_{\tau}$ get an additional term localized on the D8-brane,

$$
\begin{aligned}
\frac{1}{32 \kappa_{10}^{2}} \partial_{r}\left(\frac{r^{7}}{L^{3}} \partial_{r} C_{\tau}\right)= & 2 \pi \alpha^{\prime} \mu_{8} \partial_{r}\left(\sqrt{-g} g^{r r} g^{\tau \tau} \operatorname{Tr}\left(\mathcal{A}_{r}\right)\right) \\
& \times(\delta(\tau)+\delta(\tau-\pi)) \\
= & 2 \pi \alpha^{\prime} \mu_{8} \partial_{r}\left(\frac{r^{7}}{2^{4} L^{3}} N_{f} \hat{\mathcal{A}}_{r}\right) \\
& \times(\delta(\tau)+\delta(\tau-\pi)),
\end{aligned}
$$

which we solve by introducing the localized fluctuation $C_{\tau}^{(\delta)}$ according to

$$
\partial_{r} C_{\tau}^{(\delta)}=4 \pi \alpha^{\prime} \kappa_{10}^{2} \mu_{8} N_{f} \hat{\mathcal{A}}_{r}(\delta(\tau)+\delta(\tau-\pi)),
$$

where we have assumed $\square C_{\tau}^{(\delta)}=0$ with respect to Minkowski space coordinates and $\partial_{\mu} \hat{\mathcal{A}}^{\mu}=0$. Switching to $Z^{2}=\left(r / r_{\mathrm{KK}}\right)^{6}-1$ and with

$$
\hat{\mathcal{A}}_{Z}(Z, x)=\frac{\sqrt{2}}{\sqrt{N_{f}} \pi f_{\pi}} \frac{1}{1+Z^{2}} \eta_{0}(x),
$$


we thus obtain

$$
\begin{aligned}
C_{\tau}^{(\delta)}= & \int_{0}^{Z} d Z \partial_{Z} C_{\tau}^{(\delta)} \\
= & 4 \pi \alpha^{\prime} \kappa_{10}^{2} \mu_{8} \sqrt{N_{f}} \frac{\sqrt{2}}{\pi f_{\pi}} \arctan (Z) \eta_{0}(x) \\
& \times[\delta(\tau)+\delta(\tau-\pi)] .
\end{aligned}
$$

The "anomalous" part $C_{\tau}^{(\delta)}$ contributes to the $\theta$ parameter,

$$
\begin{aligned}
\theta & =l_{s}^{-1} \int_{S_{\tau}} C_{1}=l_{s}^{-1} \int_{\text {cigar }} F_{2}=l_{s}^{-1} \int d r d \tau \partial_{r}\left(C_{\tau}^{(0)}+C_{\tau}^{(\delta)}\right) \\
& =\vartheta(x)+\frac{2 \pi \alpha^{\prime} 4 \kappa_{10}^{2} \mu_{8} \sqrt{N_{f}}}{\sqrt{2} f_{\pi} l_{s}} \eta_{0}(x)=\vartheta(x)+\frac{\sqrt{2 N_{f}}}{f_{\pi}} \eta_{0}(x) .
\end{aligned}
$$

In the presence of flavor branes, the $\theta$ parameter is therefore no longer given by $\vartheta$ alone, but also involves $\eta_{0}$.

In the remainder of this work we will set the $\theta$ parameter to 0 , which corresponds to nonvanishing $\vartheta(x)$ according to

$$
\vartheta(x)=-\frac{\sqrt{2 N_{f}}}{f_{\pi}} \eta_{0}(x) .
$$

The meson field $\eta_{0}$ therefore also appears in the nonnormalizable mode $C_{\tau}^{(0)}$, which is a fundamental ingredient in the realization of the Witten-Veneziano mechanism in the WSS model.

Note that, in the chiral case, a constant $\theta$ can be absorbed simply in a field redefinition $\eta_{0} \rightarrow \eta_{0}+f_{\pi} \theta / \sqrt{2 N_{f}}$, since only derivatives of $\eta_{0}$ appear in the effective action produced by $S_{\mathrm{DBI}}^{\mathrm{D} 8}$. However, introducing mass terms for quarks either through world-sheet instantons or nonnormalizable modes of bifundamental fields corresponding to open-string tachyons [45-50] produces the additional term

$$
\mathcal{L}_{m}^{\mathcal{M}} \propto \int d^{4} x \operatorname{Tr}(M U(x)+\text { H.c. })
$$

where such a redefinition changes the phase of the quark mass matrix $\mathcal{M}=\operatorname{diag}\left(m_{u}, m_{d}, m_{s}\right)$ according to $\mathcal{M} \rightarrow \mathcal{M} e^{i \theta / N_{f}}$.

Both, in the chiral limit and in the case with nonzero quark masses, the singlet $\eta_{0}$ receives an extra mass term that is determined by the topological susceptibility of pure Yang-Mills theory obtained in (15),

$$
m_{0}^{2}=\frac{2 N_{f}}{f_{\pi}^{2}} \chi_{g}
$$

in accordance with the Witten-Veneziano formula $[4,5]$.

\section{PSEUDOSCALAR GLUEBALL-MESON MIXING}

With the additional term $C_{\tau}^{(\delta)} \propto \eta_{0}$ we have solved the anomalous equations of motion. ${ }^{2}$ Now we will determine the field redefinitions that are necessary to obtain a diagonal action for the Minkowski space fields $\eta_{0}$ and $\tilde{G}$. In doing this we encounter divergent terms proportional to $\delta(0)$ in analogy to the Hořava-Witten calculation [51]. By adding to the Lagrangian terms beyond the probe approximation one would presumably be able to cancel these divergences. In the following we will however just drop terms proportional to $\delta(0)$, i.e., the $\left(C_{\tau}^{(\delta)}\right)^{2}$ term in $S_{\mathrm{R}}$ and the $C_{\tau}^{(\delta)}$ term in $S_{\mathrm{CS}}$.

Let us start with the effective kinetic terms coming from the first part of (13),

$$
S_{\mathrm{R}}^{(\mathrm{kin})}=-\frac{\pi^{3}}{12 \kappa_{10}^{2} M_{\mathrm{KK}}} \int_{r_{\mathrm{KK}}}^{\infty} d r \int d^{4} x \frac{r^{3} L}{f(r)} \eta^{\mu \nu} \partial_{\mu} C_{\tau} \partial_{\nu} C_{\tau} .
$$

With $C_{\tau}=C_{\tau}^{(0)}+C_{\tau}^{(\delta)}+C_{\tau}^{(2)}$ and $C_{\tau}^{(2)}(r, x) \propto \tilde{G}(x)$ we write them as

$S_{\mathrm{R}}^{(\text {kin })}=\int d^{4} x\left(\zeta_{1} \partial_{\mu} \eta_{0} \partial^{\mu} \eta_{0}+\zeta_{2} \partial_{\mu} \eta_{0} \partial^{\mu} \tilde{G}-\frac{1}{2} \partial_{\mu} \tilde{G} \partial^{\mu} \tilde{G}\right)$,

where we have fixed the normalization of the radial mode functions in $C_{\tau}^{(2)}$ by requiring

$$
\frac{\pi^{3}}{12 \kappa_{10}^{2} M_{\mathrm{KK}}} \int_{r_{\mathrm{KK}}}^{\infty} d r \frac{r^{3} L}{f(r)}\left(C_{\tau}^{(2)} / \tilde{G}\right)^{2}=\frac{1}{2} .
$$

For the constant $\zeta_{1}$ which corresponds to a wave function renormalization of $\eta_{0}$ we obtain

$$
\begin{aligned}
\zeta_{1} & =-\frac{\pi^{3}}{12 \kappa_{10}^{2} M_{\mathrm{KK}}} \int_{r_{\mathrm{KK}}}^{\infty} d r \frac{r^{3} L}{f(r)}\left(C_{\tau}^{(0)}+2 C_{\tau}^{(\delta)}\right) C_{\tau}^{(0)} / \eta_{0}^{2} \\
& =: \frac{N_{f}}{N_{c}} \bar{\zeta}_{1},
\end{aligned}
$$

where $\bar{\zeta}_{1}$ is divergent, since it is obtained by integrating non-normalizable modes, but $\zeta_{1}$ is suppressed by a factor $N_{c} / N_{f}$ compared to the kinetic term for $\eta_{0}$ contained in $S_{\mathrm{DBI}}^{\mathrm{D} 8}$. The constant $\zeta_{2}$ which is associated with a kinetic mixing of $\eta_{0}$ and pseudoscalar glueball modes is finite and given by

\footnotetext{
${ }^{2}$ As stated after (22), we had to assume $\square C_{\tau}^{(\delta)}=0$ with respect to Minkowski coordinates. With $\eta_{0}$ picking up the mass $m_{0}^{2}$, this assumption is violated, but only at higher order in $N_{f}$ : $\square C_{\tau}^{(\delta)} \sim N_{f}^{1 / 2} \square \eta_{0} \propto N_{f}^{3 / 2}$. (With nonzero quark masses, this seems safe as long as their contributions to the masses of the pseudoscalar mesons are much smaller than $m_{0}$.)
} 


$$
\begin{aligned}
\zeta_{2} & =-\frac{\pi^{3}}{12 \kappa_{10}^{2} M_{\mathrm{KK}}} \int_{r_{\mathrm{KK}}}^{\infty} d r \frac{r^{3} L}{f(r)}\left(C_{\tau}^{(0)}+C_{\tau}^{(\delta)}\right) C_{\tau}^{(2)} /\left(\eta_{0} \tilde{G}\right) \\
& =0.011180 \cdots \sqrt{\frac{N_{f}}{N_{c}} \lambda}
\end{aligned}
$$

for the lowest pseudoscalar glueball. (The next-to-lightest, excited pseudoscalar glueball has $\zeta_{2}=-0.014314 \ldots$ $\sqrt{N_{f} / N_{c}} \lambda$.)

The remaining terms from the background action (13) and the CS action (20)

$$
\begin{aligned}
S_{\mathrm{R}}^{(\mathrm{mass})}= & -\frac{1}{4 \kappa_{10}^{2}} \int d^{10} x \sqrt{g_{S^{4}}} \frac{1}{2^{4}} \frac{r^{7}}{L^{3}}\left(\partial_{r} C_{\tau}\right)^{2} \\
& +\mu_{8} 2 \pi \alpha^{\prime} \int d^{10} x(\delta(\tau)+\delta(\tau-\pi)) \\
& \cdot \sqrt{\left|g_{10}\right|} \operatorname{Tr}\left(\mathcal{A}_{r}\right) g^{r r} g^{\tau \tau} \partial_{r} C_{\tau}
\end{aligned}
$$

give the effective mass terms

$$
S_{\mathrm{R}}^{(\mathrm{mass})}=-\frac{1}{2} m_{0}^{2} \eta_{0}^{2}-\frac{1}{2} M_{G}^{2} \tilde{G}^{2}+\zeta_{3} \eta_{0} \tilde{G},
$$

with the values already determined above,

$$
m_{0}^{2}=\frac{\pi V_{4}}{\kappa_{10}^{2} M_{\mathrm{KK}}} \int_{r_{\mathrm{KK}}}^{\infty} d r \frac{1}{2^{4}} \frac{r^{7}}{L^{3}}\left(\partial_{r} C_{\tau}^{(0)} / \eta_{0}\right)^{2}=\frac{\lambda^{2} N_{f}}{27 \pi^{2} N_{c}} M_{\mathrm{KK}}^{2}
$$

and (for the lightest pseudoscalar glueball mode)

$$
M_{G}^{2}=\frac{\pi V_{4}}{\kappa_{10}^{2} M_{\mathrm{KK}}} \int_{r_{\mathrm{KK}}}^{\infty} d r \frac{1}{2^{4}} \frac{r^{7}}{L^{3}}\left(\partial_{r} C_{\tau}^{(2)} / \tilde{G}\right)^{2}=3.5539 M_{\mathrm{KK}}^{2}
$$

The terms involving $\partial_{r} C_{\tau}^{(\delta)} \partial_{r}\left[C_{\tau}^{(0)}+C_{\tau}^{(2)}\right]$ cancel by the equation of motion (22) with the CS term. The mass mixing term $\eta_{0} \tilde{G}$ vanishes because

$$
\zeta_{3} \propto \int_{r_{\mathrm{KK}}}^{\infty} d r r^{7} \partial_{r} C_{\tau}^{(0)} \partial_{r} C_{\tau}^{(2)} \propto \int_{r_{\mathrm{KK}}}^{\infty} d r \partial_{r} C_{\tau}^{(2)}=0
$$

as already pointed out in [14].

However, the term involving $C_{\tau}^{(\delta)} C_{\tau}^{(2)}$ in (33) has produced a nontrivial kinetic mixing term of order $\left(N_{f} / N_{c}\right)^{1 / 2}$. To get rid of such mixing terms one has to perform a nonunitary field redefinition. In the present case, one has to make the substitutions

$$
\begin{aligned}
\eta_{0} & \rightarrow\left(1+\zeta_{1}\right) \eta_{0}+\zeta_{2} \tilde{G}, \\
\tilde{G} & \rightarrow\left(1+\frac{1}{2} \zeta_{2}^{2}\right) \tilde{G},
\end{aligned}
$$

which yield

$$
\begin{aligned}
\mathcal{L}_{\eta_{0}, \tilde{G}}^{\mathrm{bilin}}= & -\frac{1}{2}\left(\partial_{\mu} \eta_{0}\right)^{2}-\frac{1}{2}\left(\partial_{\mu} \tilde{G}\right)^{2} \\
& +\partial_{\mu} \eta_{0} \partial^{\mu} \tilde{G}\left(2 \zeta_{1} \zeta_{2}+\frac{1}{2} \zeta_{2}^{3}\right)-\frac{1}{2} M_{G}^{2}\left(1+\zeta_{2}^{2}\right) \tilde{G}^{2} \\
& -\frac{1}{2} m_{0}^{2} \eta_{0}^{2}-m_{0}^{2} \zeta_{2} \eta_{0} \tilde{G}+O\left(N_{f}^{2} / N_{c}^{2}\right) \\
= & -\frac{1}{2}\left(\partial_{\mu} \eta_{0}\right)^{2}-\frac{1}{2}\left(\partial_{\mu} \tilde{G}\right)^{2}-\frac{1}{2} M_{G}^{2}\left(1+\zeta_{2}^{2}\right) \tilde{G}^{2} \\
& -\frac{1}{2} m_{0}^{2} \eta_{0}^{2}+O\left(N_{f}^{3 / 2} / N_{c}^{3 / 2}\right)
\end{aligned}
$$

where we have taken into account that $\zeta_{2} \propto \sqrt{N_{f} / N_{c}}$ while $\zeta_{1}, m_{0}^{2} \propto N_{f} / N_{c}$. The field redefinitions (39) thus diagonalize the bilinear terms up to and including order $N_{f} / N_{c}$, yielding also a small positive contribution to the pseudoscalar glueball mass term,

$$
\begin{aligned}
M_{G}^{2} & =(1789.0 \mathrm{MeV})^{2} \rightarrow\left(1+(0.01118 \lambda)^{2} N_{f} / N_{c}\right) M_{G}^{2} \\
& =(1819.7 \ldots 1806.5 \mathrm{MeV})^{2}
\end{aligned}
$$

for $\lambda=16.63 \ldots 12.55$.

Dropping all terms of order $\left(N_{f} / N_{c}\right)^{3 / 2}$ and higher, we see that the divergent coefficient $\zeta_{1}$ can be ignored and that (39) reduces to

$$
\begin{aligned}
& \eta_{0} \rightarrow \eta_{0}+\zeta_{2} \tilde{G}=\eta_{0}+0.01118 \sqrt{\frac{N_{f}}{N_{c}}} \lambda \tilde{G}, \\
& \tilde{G} \rightarrow \tilde{G} .
\end{aligned}
$$

Note that this is very different from the mixing scenario proposed by Rosenzweig et al. in [20,21] and also considered in [25]. In that scenario it is assumed that the chiral anomaly is not saturated by $\eta_{0}$ alone, but only together with the pseudoscalar glueball field. ${ }^{3}$ This leads to a nondiagonal mass matrix for $\eta_{0}$ and the pseudoscalar glueball which can be diagonalized by an orthogonal matrix. The field redefinition (39), on the other hand, is nonunitary. It is needed to remove the kinetic mixing term (30), but originally there is no mass mixing term because of (38); a mass mixing term appears after the field redefinition in (40), but only at the order $N_{f}^{3 / 2} / N_{c}^{3 / 2}$ which is beyond the probe approximation. ${ }^{4}$

\footnotetext{
${ }^{3}$ According to [20], this additional field could however also be interpreted as a radial excitation of $\eta^{\prime}$.

${ }^{4}$ In $[22,25,26]$ a unitary mixing of $\eta^{\prime}$ and pseudoscalar glueballs has been considered from a phenomenological point of view, which has an important impact on the determination of the pseudoscalar mixing angle $\theta_{P}$ when there is significant $\eta^{\prime}-\tilde{G}$ mixing. By contrast, the nonunitary transformation (42) that we obtained does not have this effect (to leading order).
} 
In Ref. [14], where (in view of the vanishing mass mixing term) the interactions of the unmixed pseudoscalar glueball were considered, it was found that those are given either by pairs of pseudoscalar glueballs interacting with scalar or tensor glueballs or by a vertex connecting a pseudoscalar glueball with $\eta_{0}$ and a scalar glueball. The latter, which is relevant for the decay of a pseudoscalar glueball, is due to the fact that the integral (38) for the $\eta_{0} \tilde{G}$ mass mixing term no longer vanishes when metric fluctuations dual to a scalar glueball are inserted. Assuming that this is the dominant vertex, a very narrow pseudoscalar glueball was predicted whose decays had to involve $\eta$ or $\eta^{\prime}$ together with the decay products of a scalar glueball.

Through the shift (42) a pseudoscalar glueball acquires also all types of vertices that the singlet $\eta_{0}$ possesses. In the DBI part of the D8-brane action, such vertices are contained only in terms involving $\mathcal{F}$ to fourth and higher power, which are suppressed by higher powers of $\alpha^{\prime}$. In the chiral limit, the dominant interactions come from the CS part of the D8-brane. With quark masses introduced according to (27), further interactions arise from (assuming $\left.\mathcal{M}=\mathcal{M}^{\dagger}\right)$

$$
\begin{aligned}
\operatorname{Tr}\left(\mathcal{M}\left(U+U^{\dagger}\right)\right) \rightarrow & \operatorname{Tr}\left(\mathcal{M}\left(U+U^{\dagger}\right)\right) \\
& +i \zeta_{2} f_{\pi}^{-1} \sqrt{2 / N_{f}} \tilde{G} \operatorname{Tr}\left(\mathcal{M}\left(U-U^{\dagger}\right)\right) .
\end{aligned}
$$

The additional term in (43) also contains a bilinear term involving $\tilde{G}$ and $\eta_{0,8}$,

$$
\Delta \mathcal{L}_{m}^{(2)}=-\frac{2 \zeta_{2}}{\sqrt{3}} \tilde{G}\left[\frac{1}{\sqrt{3}}\left(m_{K}^{2}+\frac{1}{2} m_{\pi}^{2}\right) \eta_{0}-\sqrt{\frac{2}{3}}\left(m_{K}^{2}-m_{\pi}^{2}\right) \eta_{8}\right] .
$$

It implies a further mixing of $\tilde{G}$ with $\eta_{0,8}$ which turns out to be negligible as concerns the masses of $\eta, \eta^{\prime}$, and $\tilde{G}$ when the mass matrix of the $\tilde{G}-\eta_{0}-\eta_{8}$ sector is diagonalized. Due to the large mass of the pseudoscalar glueball, the corrections to the masses of $\eta, \eta^{\prime}$, and $\tilde{G}$ are only about $-0.015 \%$, $-0.008 \%$, and $+0.09 \%$, respectively, and thus can be safely neglected. Also the vertices induced by the kinetic mixing of $\tilde{G}$ and $\eta_{0}$ receive only small corrections of the order $\zeta_{2} m_{K}^{2} / m_{G}^{2} \ll \zeta_{2}$. However, the small mixing of $\tilde{G}$ with $\eta_{8}$ gives rise to new types of vertices from the DBI action.

In the next section we shall consider the consequences of all these additional interactions in turn.

\section{PSEUDOSCALAR GLUEBALL DECAY MODES}

\section{A. Decay into two vector mesons}

The dominant decay channel of the pseudoscalar glueball turns out to be a decay into two vector mesons. This
TABLE I. Partial decay widths in $\mathrm{MeV}$ for the decay of a pseudoscalar glueball in two vector mesons with $\lambda=$ $16.63 \ldots 12.55$ and $M_{G}$ given by the WSS result (41) and also when extrapolated to the lattice prediction of $2600 \mathrm{MeV}$ with reduced mixing (see text).

\begin{tabular}{lcc}
\hline \hline & $\Gamma_{p}\left(M_{G}=1813 \pm 7 \mathrm{MeV}\right)$ & $\Gamma_{p}\left(M_{G}=2600 \mathrm{MeV}\right)$ \\
\hline$\rho \rho$ & $36.8 \ldots 45.0$ & $190 \ldots 248$ \\
$\omega \omega$ & $11.4 \ldots 13.8$ & $62 \ldots 81$ \\
$K^{*} \bar{K}^{*}$ & $2.7 \ldots 1.8$ & $189 \ldots 246$ \\
$\phi \phi$ & & $29 \ldots 38$ \\
$a_{1} a_{1}$ & $3.1 \ldots 4.0$ \\
\hline$\sum_{v v}$ & $51 \ldots 61$ & $473 \ldots 618$ \\
\hline \hline
\end{tabular}

decay mode arises from the Chern-Simons term and is mediated by the mixing of $\eta_{0}$ with the glueball $\tilde{G}$ according to (42). Before the field redefinition we have the interaction term

$$
\begin{aligned}
S_{\mathrm{CS}}^{\mathrm{D} 8} & \supset \mu_{8} \frac{\left(2 \pi \alpha^{\prime}\right)^{3}}{3 !} \int \operatorname{Tr}\left(\mathcal{F} \wedge \mathcal{F} \wedge \mathcal{F} \wedge C_{3}\right) \\
& \supset \mu_{8} \frac{\left(2 \pi \alpha^{\prime}\right)^{3}}{2 g_{s}} L^{3} \pi^{2} \epsilon^{\mu \nu \rho \sigma} \int \operatorname{Tr}\left(\hat{\mathcal{A}}_{Z} \partial_{\mu} \mathcal{A}_{\nu} \partial_{\rho} \mathcal{A}_{\sigma}\right),
\end{aligned}
$$

with $\hat{\mathcal{A}}_{Z} \propto \eta_{0}$, which after the field redefinition yields the term

$$
S_{\mathrm{CS}}^{\mathrm{D} 8} \supset k_{1} \tilde{G} \epsilon^{\mu \nu \rho \sigma} \operatorname{Tr} \partial_{\mu} v_{\nu} \partial_{\rho} v_{\sigma}
$$

with the coupling constant

$$
\begin{aligned}
k_{1} & =\zeta_{2} \frac{1}{\sqrt{N_{f}}} U_{\mathrm{KK}} \mu_{8} \frac{\left(2 \pi \alpha^{\prime}\right)^{3}}{2 g_{s}} L^{3} \pi^{2} \int d Z \phi_{0} \psi_{1}^{2} \\
& =\zeta_{2} \times 877.39 \lambda^{-3 / 2} N_{f}^{-1 / 2} N_{c}^{-1 / 2} M_{\mathrm{KK}}^{-1} \\
& =9.8092 M_{\mathrm{KK}}^{-1} N_{c}^{-1} \lambda^{-\frac{1}{2}},
\end{aligned}
$$

for the lowest vector meson mode $\psi_{1}$. For the lowest axialvector mesons with radial mode function $\psi_{2}$ and mass square eigenvalue $m_{a_{1}}^{2} \approx 1.57 M_{\mathrm{KK}}^{2} \approx(1190 \mathrm{MeV})^{2}$, which is quite close to the experimental value of $1230 \mathrm{MeV}$ of the lightest $a_{1}$ axial-vector meson, ${ }^{5}$ we obtain instead $k_{2}=4.8888 M_{\mathrm{KK}}^{-1} N_{c}^{-1} \lambda^{-\frac{1}{2}}$.

Performing the polarization sums we obtain the amplitude squared

$$
\sum_{\epsilon_{1}, \epsilon_{2}}|\mathcal{M}(\tilde{G} \rightarrow v v)|^{2}=8 k_{1}^{2} M^{2}\left(\frac{M^{2}}{4}-m^{2}\right),
$$

\footnotetext{
${ }^{5}$ In Table I we ignore the slightly heavier $f_{1}$ and $K_{1}$ axialvector mesons, which would start contributing significantly only for $M_{G}$ well above $2600 \mathrm{MeV}$.
} 
TABLE II. Partial decay widths in MeV for the decay of a pseudoscalar glueball in three pseudoscalar mesons resulting from (49) with $\lambda=16.63 \ldots 12.55$. The results for $\tilde{G} \rightarrow K \bar{K} \pi$ do not yet include the decays $\tilde{G} \rightarrow K K^{*} \rightarrow K \bar{K} \pi$ ) discussed in Sec. IV C, and those for $\tilde{G} \rightarrow P P \eta^{\left({ }^{\prime}\right)}$ with $P$ a pseudoscalar meson neglect $\tilde{G} \rightarrow f_{0}(1710) \eta^{\left({ }^{\prime}\right)} \rightarrow P P \eta^{(')}$. For comparison, the decay modes for an unmixed pseudoscalar glueball via $\tilde{G} \rightarrow$ $f_{0}(1710) \eta\left({ }^{\prime}\right)$ as obtained in Ref. [14] are evaluated in the last entry of the table for the same set of parameters. These latter results are however incomplete in the mixed case (see text).

\begin{tabular}{lcc}
\hline \hline & $\Gamma_{p}\left(M_{G}=1813 \pm 7 \mathrm{MeV}\right)$ & $\Gamma_{p}\left(M_{G}=2600 \mathrm{MeV}\right)$ \\
\hline$K \bar{K} \pi\left(\right.$ w/o $\left.K K^{*}\right)$ & $0.398 \ldots 0.387$ & $0.558 \ldots 0.550$ \\
\hline$K \bar{K} \eta\left(\right.$ w/o $f_{0}(1710) \eta$ etc. $)$ & $0.0263 \ldots 0.0064$ & $0.1092 \ldots 0.0285$ \\
$K \bar{K} \eta^{\prime}$ & & $0.3303 \ldots 0.3570$ \\
$\pi \pi \eta$ & $0.0048 \ldots 0.0061$ & $0.0049 \ldots 0.0061$ \\
$\pi \pi \eta^{\prime}$ & $0.0011 \ldots 0.0007$ & $0.0021 \ldots 0.0013$ \\
$\eta \eta \eta$ & $0.0039 \ldots 0.0007$ & $0.0337 \ldots 0.0064$ \\
$\eta \eta \eta^{\prime}$ & & $0.0564 \ldots 0.0277$ \\
$\eta \eta^{\prime} \eta^{\prime}$ & & $0.0047 \ldots 0.0048$ \\
\hline$P P \eta^{\left({ }^{\prime}\right)}\left(\right.$ w/o $\left.f_{0}(1710) \eta^{\left({ }^{\prime}\right)}\right)$ & $0.036 \ldots 0.014$ & $0.540 \ldots 0.431$ \\
\hline$f_{0}(1710) \eta^{\left({ }^{\prime}\right)} \rightarrow P P \eta^{\left({ }^{\prime}\right)}[14]$ & $0.0068 \ldots 0.015$ & $2.5 \ldots 4.3$ \\
\hline \hline
\end{tabular}

where $M$ and $m$ are the masses of $\tilde{G}$ and $v$ (and similarly for the heavier vector and axial-vector modes when $M$ is large enough).

The partial widths for decays into the various vector mesons are listed in Table I for $\lambda=16.63 \ldots 12.55$ and glueball mass $M_{G}$ given alternatively by the WSS result (41) and by an (admittedly speculative) extrapolation to the lattice prediction of $2600 \mathrm{MeV}$, where we have assumed that the mixing parameter $\zeta_{2}$ and thus $k_{1}$ (which has inverse mass dimension) scales like $M_{G}^{-1}$ when the glueball mass is raised. (Keeping the mixing parameter as is, the widths for a $2600 \mathrm{MeV}$ glueball would all be a factor of about 2 larger.) Also with the assumed reduction of $k_{1}$, a $2600 \mathrm{MeV}$ pseudoscalar glueball is thus projected to be a rather broad resonance.

\section{B. Decay into three pseudoscalar mesons}

In Ref. [52], Gounaris and Neufeld have proposed that a pure pseudoscalar glueball decays predominantly through an interaction Lagrangian involving $i \tilde{G} \operatorname{Tr}\left(M\left(U-U^{\dagger}\right)\right)$ in order to explain that the pseudoscalar glueball candidate at the time, $\imath(1460){ }^{6}$ seemed to decay mainly into $K \bar{K} \pi$. Decays into $\eta \pi \pi$ would thus be suppressed by a factor $\left(m_{\pi} / m_{K}\right)^{4}$.

In Sec. III we have found that such an interaction Lagrangian is in fact generated in the WSS model with nonzero quark masses by the kinetic mixing of $\eta_{0}$ and pseudoscalar glueball modes. Explicitly, it reads

\footnotetext{
${ }^{6}$ This is now listed as two states $\eta(1405)$ and $\eta(1475)$ by the Data Particle Group [53], of which the lighter one is still considered occasionally as a glueball candidate [37] in view of its supernumerary nature with respect to the quark model, while others question the existence of two separate states and argue for a single $\eta(1440)$ [36].
}

$$
\Delta \mathcal{L}_{m}=i \zeta_{2} \frac{f_{\pi}}{2 \sqrt{2 N_{f}}} \tilde{G} \operatorname{Tr} \overline{\mathcal{M}}\left(U-U^{\dagger}\right)
$$

with

$$
\overline{\mathcal{M}}=\operatorname{diag}\left(m_{\pi}^{2}, m_{\pi}^{2}, 2 m_{K}^{2}-m_{\pi}^{2}\right)
$$

in the isospin symmetric case $m_{u}=m_{d}$. This contains vertices of the pseudoscalar glueball with $K \bar{K} \pi, K \bar{K} \eta\left({ }^{\prime}\right)$, and $\eta\left({ }^{\prime}\right)^{3}$ of the order of $\zeta_{2} m_{K}^{2} / f_{\pi}^{2}$, and vertices with $\eta\left({ }^{\prime}\right) \pi \pi$ proportional to $\zeta_{2} m_{\pi}^{2} / f_{\pi}^{2}$.

In Table II we list the resulting partial decay widths for a pseudoscalar glueball with mass $M=1813 \pm 7 \mathrm{MeV}$ according to (41) and, as above, with mass extrapolated to $2600 \mathrm{MeV}$ as predicted by quenched lattice QCD.

In chiral perturbation theory, a well-known problem is that the leading-order Lagrangian severely underestimates the decay $\eta^{\prime} \rightarrow \eta \pi \pi$ whose leading-order amplitude is proportional to $m_{\pi}^{2}$, to wit, $\left|\mathcal{M}\left(\eta^{\prime} \rightarrow \eta \pi \pi\right)\right|=$ $\left|2 \sqrt{2} \cos \left(2 \theta_{P}\right)-\sin \left(2 \theta_{P}\right)\right|\left(m_{\pi} / f_{\pi}\right)^{2} / 6$. This accounts for only about $3 \%$ of the experimental result. Higher-order terms in chiral perturbation theory which are not present in the WSS model have been shown to give contributions which are much larger [54,55]. By the same token, the partial decay rates of a pseudoscalar glueball into three pseudoscalar mesons that result from its mixing with $\eta_{0}$ can be expected to be strongly underestimated by (49), in particular the amplitudes for decay into $\eta \pi \pi$, which are proportional to $m_{\pi}^{2}$. The other decay amplitudes are of the order of $m_{K}^{2}$, but since $m_{K}$ is small compared to $m_{G}$, formally higher-order contributions could again be much more important. However, even if we assume that these partial decay widths are underestimated by an order of magnitude, they are still much smaller than the decays into two vector mesons. 
In Ref. [14], one of us with F. Brünner has calculated the decays of an unmixed pseudoscalar glueball into three pseudoscalar mesons in the WSS model, which necessarily involves $\eta_{0}$ and a scalar glueball. If the latter is identified with $f_{0}(1710)$, which decays predominantly into kaons (as is predicted $[30,31]$ by the WSS model when scalar glueballs have no or negligible decay width into $\left.\eta \eta^{\prime}\right)$, the dominant channel is $\tilde{G} \rightarrow f_{0}(1710) \eta \rightarrow K \bar{K} \eta$, whereas $K K \pi$ decays are excluded. With mixing, additional amplitudes for $\tilde{G} \rightarrow P P \eta\left({ }^{\prime}\right)$ arise from $G_{\text {scalar }} \eta_{0}^{2}$ and $G_{\text {scalar,tensor }}\left(\partial_{\mu} \eta_{0}\right)^{2}$ terms in the DBI action and also from the additional terms involving $C_{\tau}^{(\delta)}$ in $S_{R}$. They would have to be included in a complete calculation of $P P \eta\left({ }^{\prime}\right)$ decays. To give an idea of the magnitude of the contributions from $\tilde{G} \rightarrow f_{0}(1710) \eta\left({ }^{\prime}\right) \rightarrow P P \eta\left({ }^{\prime}\right)$, in Table II we have included the partial widths of these decay modes as obtained in the unmixed case in [14]. While such contributions are moderate for the pseudoscalar glueball mass (41), they become important for $M_{G}$ extrapolated to the mass predicted by lattice which is well above the threshold for $f_{0}(1710)+\eta$ decays. However, also these contributions are always much smaller than those for the decays $\tilde{G} \rightarrow v v$.

\section{Decay into one pseudoscalar meson together with one or two vector mesons}

The very small mixing of a pseudoscalar glueball with $\eta_{8}$ that is induced by the quark mass term (44) also gives rise to vertices with one pseudoscalar meson and one or two vector mesons due to the terms involving $\operatorname{Tr}\left(\partial_{\mu} \Pi,\left[V^{\mu}, \Pi\right]\right)$ and $\operatorname{Tr}\left(\left[V_{\mu}, \Pi\right]\left[V^{\mu}, \Pi\right]\right)$ in the Yang-Mills part of the effective action. When the glueball mass is above the respective threshold, this gives rise to decay modes

$\tilde{G} \rightarrow K K^{*}, \pi K^{*} K^{*}, K K^{*} \rho, K K^{*} \omega, K K^{*} \phi, \eta\left({ }^{\prime}\right) K^{*} K^{*}$,

where $K K^{*}$ is short for $\bar{K} K^{*}, K \bar{K}^{*}$ etc. The corresponding amplitudes are proportional to $\zeta_{2}\left(m_{K}^{2}-m_{\pi}^{2}\right) / m_{G}^{2} \sim 10^{-2}$ times a factor $g_{\rho \pi \pi} \propto \lambda^{-1 / 2} N_{c}^{-1 / 2}$ or $g_{\rho \rho \pi \pi} \propto \lambda^{-1} N_{c}^{-1}$.

TABLE III. Partial decay widths in $\mathrm{MeV}$ for the decay of a pseudoscalar glueball into one pseudoscalar meson and one or two vector mesons.

\begin{tabular}{lcc}
\hline \hline & $\Gamma_{p}\left(M_{G}=1813 \pm 7 \mathrm{MeV}\right)$ & $\Gamma_{p}\left(M_{G}=2600 \mathrm{MeV}\right)$ \\
\hline$K K^{*}$ & $0.381 \ldots 0.288$ & $0.302 \ldots 0.225$ \\
\hline$\pi K^{*} K^{*}$ & & $0.0113 \ldots 0.0112$ \\
$K K^{*} \rho$ & $(2.50 \ldots .2 .47) \times 10^{-3}$ \\
$K K^{*} \omega$ & $(0.799 \ldots 0.787) \times 10^{-3}$ \\
$K K^{*} \phi$ & $(0.253 \ldots 0.249) \times 10^{-3}$ \\
$\eta K^{*} K^{*}$ & $(0.097 \ldots 0.026) \times 10^{-3}$ \\
\hline \hline
\end{tabular}

In Table III the resulting partial decay widths are listed. While the partial widths for decays of the pseudoscalar glueball into $K^{*}$, one further vector meson and one pseudoscalar meson are rather small, the partial width for $\tilde{G} \rightarrow K K^{*}$ (and subsequently $\rightarrow K K \pi$ ) turns out to be comparable to that of the direct decay $\tilde{G} \rightarrow K K \pi$ evaluated above. Since we assume that the latter may be underestimated significantly, we have not carried out a full calculation of $\tilde{G} \rightarrow K K \pi$ combining coherently both amplitudes.

In summary, we are finding a rich pattern of decays of the pseudoscalar glueball in three or more mesons, which involve vertices which are proportional to pseudoscalar meson masses. The by far dominant decay modes are however given by two vector mesons.

\section{DISCUSSION AND OUTLOOK}

If the mass of the pseudoscalar glueball is as given by the WSS model, (41), the pseudoscalar glueball is predicted to be a rather narrow resonance with relative width $\Gamma / M \approx 0.03 \ldots 0.04$. However, the WSS model appears to underestimate significantly the mass of heavier glueballs. The tensor glueball comes out as $1487 \mathrm{MeV}$, while it is predicated as around $2400 \mathrm{MeV}$ by both quenched and unquenched lattice QCD [28,29,33-35]. Extrapolating our results to a significantly higher pseudoscalar glueball mass as indicated by (quenched) lattice QCD, $2.6 \mathrm{GeV}$, the prediction is instead that of a very broad resonance. In Fig. 1 we display two possible extrapolations, one with unchanged vertices and mixing when the glueball mass is increased in the final formulas, which leads to an extremely large total width, and the somewhat more moderate scenario (underlying the above tables) where the mixing parameter $\zeta_{2}$ is assumed to be

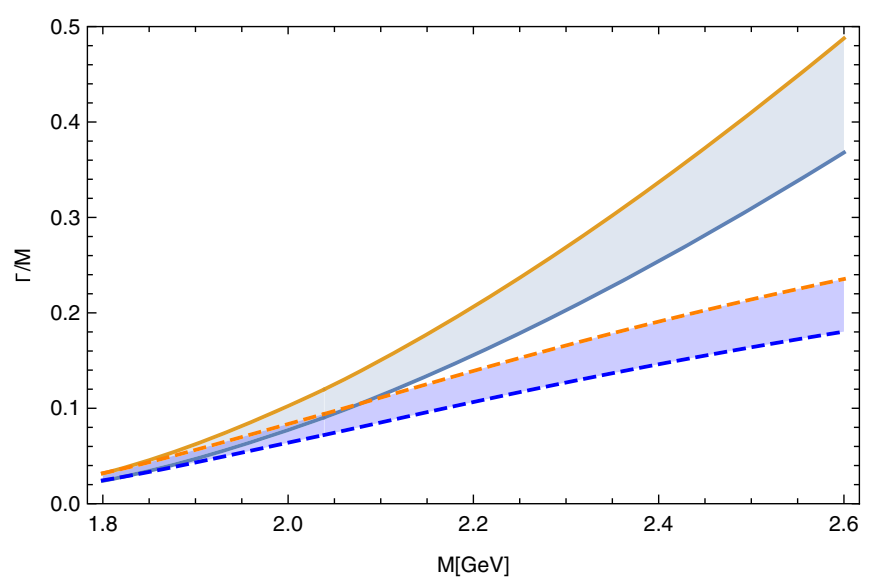

FIG. 1. Relative pseudoscalar glueball decay width $\Gamma / M$ for $\lambda=16.63$ (blue) and 12.55 (orange) with two different extrapolations to larger masses: same vertices and mixing (full lines) or when the mixing parameter $\zeta_{2}$ is assumed to be decreasing like $M_{\mathrm{WSS}} / M$ (dashed lines). 
decreasing like $M_{\mathrm{WSS}} / M$. (The latter extrapolation could also be interpreted as a corresponding increase of the mass parameter $M_{\mathrm{KK}}$ which sets the scale of the glueball masses. ${ }^{7}$ )

The rather large decay width of a pseudoscalar glueball to two vector mesons that we obtained from the WSS model also implies a significant coupling to photons because of the vector meson dominance that is inherent in the WSS model [10]. This also applies to the scalar and tensor glueballs studied before in [32]. Radiative glueball decays will be studied in detail in forthcoming work, as well as the contribution of glueballs to hadronic light-bylight scattering, which is of relevance to the theoretical prediction for the muon $g-2$, where holographic QCD has been argued to provide a promising framework for quantitative predictions [60].

An important implication of the mixing-induced vertices determined in this work is that contrary to the unmixed case [32] the WSS model predicts sizeable vertices for the production of single pseudoscalar glueballs in double diffractive scattering. While Regge regime hadronic scattering requires extensions and extrapolations of the WSS model [61-63], the structure of the vertices with Reggeons and Pomerons are completely determined by the Chern-Simons action and the mixing of the pseudoscalar glueball with $\eta_{0}$. For Reggeons, the vertices have the form (46), while

\footnotetext{
${ }^{7}$ The standard choice $[9,10] M_{\mathrm{KK}}=949 \mathrm{MeV}$ corresponds to using the $\rho$ meson mass as input, which leads to a good match of hadronic decay widths of $\rho$ and $\omega$ mesons for the range of $\lambda$ considered here [32]. Lattice results for the glueball spectrum clearly favor larger values of $M_{\mathrm{KK}}$. The early studies of baryons in the WSS model $[56,57]$ instead seemed to require $M_{\mathrm{KK}}$ much smaller than $949 \mathrm{MeV}$ [57], but more refined treatments [58,59] have found a good fit of baryon masses with the standard choice of $949 \mathrm{MeV}$.
}

Pomeron vertices can be derived from the A-roof genus factor in (19) as in [61]. This leads to ${ }^{8}$

$$
\begin{aligned}
\mathcal{L}_{\mathrm{CS}} \supset \zeta_{2} \tilde{G} \epsilon^{\mu \nu \rho \sigma}\left[\kappa_{a} \partial_{\nu} \mathbb{P}_{\alpha \mu} \partial_{\sigma} \mathbb{P}_{\rho}^{\alpha}\right. \\
\left.+\kappa_{b} \epsilon^{\mu \nu \rho \sigma} \partial_{\nu} \partial^{\alpha} \mathbb{P}_{\mu \beta}\left(\partial_{\sigma} \partial^{\beta} \mathbb{P}_{\alpha \rho}-\partial_{\sigma} \partial_{\alpha} \mathbb{P}_{\rho}^{\beta}\right)\right]
\end{aligned}
$$

with $\zeta_{2} \kappa_{a} M_{\mathrm{KK}}, \zeta_{2} \kappa_{b} M_{\mathrm{KK}}^{3} \propto \lambda^{-1 / 2} N_{c}^{-2}$. The results for the unmixed case of Ref. [32] instead implied that central exclusive production of pseudoscalar glueballs required the formation of pairs $\tilde{G} \tilde{G}, \eta\left({ }^{\prime}\right) \tilde{G}$, or $G_{\text {scalar,tensor }} \tilde{G}$ with vertices of order $\lambda^{-1} N_{c}^{-2}, N_{f}^{1 / 2} N_{c}^{-5 / 2}$, or $\lambda^{-1} N_{f} N_{c}^{-3}$, respectively. The possibility of production of single pseudoscalar glueballs of course lowers the threshold significantly.

Similarly, the kinetic mixing of the pseudoscalar glueball with $\eta_{0}$ allows the production of single pseudoscalar glueballs in radiative $J / \psi$ decays with rates of the parametric order $N_{c}^{-1}$ times that of the decay rate for $J / \psi \rightarrow \gamma \eta_{c}$.

However, the (semi-)quantitative results that we obtained within the WSS model suggest that the experimental identification of the pseudoscalar glueball may be made difficult by a very large decay width if its mass is around $2.6 \mathrm{GeV}$ as indicated by (quenched) lattice results. In future work we intend to further explore these predictions in the context of radiative $J / \psi$ decays and central exclusive production in the Regge regime.

\section{ACKNOWLEDGMENTS}

We thank Francesco Bigazzi for useful discussions. J. L. has been supported by the doctoral programme DKPI of the Austrian Science Fund FWF, Project No. W1252-N27.

\footnotetext{
${ }^{8} \mathrm{Up}$ to partial integrations, the tensorial structure of the two terms in (52) is in fact in one-to-one correspondence to the two couplings $g_{\mathbb{P P} \tilde{M}}^{\prime}$ and $g_{\mathbb{P P} \tilde{M}}^{\prime \prime}$ for two tensor pomerons with one pseudoscalar meson in [64].
}

[1] S. Weinberg, The U(1) problem, Phys. Rev. D 11, 3583 (1975).

[2] G. 't Hooft, Symmetry Breaking through Bell-Jackiw Anomalies, Phys. Rev. Lett. 37, 8 (1976).

[3] G. 't Hooft, How instantons solve the U(1) problem, Phys. Rep. 142, 357 (1986).

[4] G. Veneziano, U(1) without instantons, Nucl. Phys. B159, 213 (1979).

[5] E. Witten, Current algebra theorems for the U(1) "Goldstone Boson", Nucl. Phys. B156, 269 (1979).

[6] A. Armoni, Witten-Veneziano from Green-Schwarz, J. High Energy Phys. 06 (2004) 019.

[7] J. L. Barbon, C. Hoyos-Badajoz, D. Mateos, and R. C. Myers, The holographic life of the $\eta^{\prime}$, J. High Energy Phys. 10 (2004) 029.
[8] E. Witten, Anti-de Sitter space, thermal phase transition, and confinement in gauge theories, Adv. Theor. Math. Phys. 2, 505 (1998).

[9] T. Sakai and S. Sugimoto, Low energy hadron physics in holographic QCD, Prog. Theor. Phys. 113, 843 (2005).

[10] T. Sakai and S. Sugimoto, More on a holographic dual of QCD, Prog. Theor. Phys. 114, 1083 (2005).

[11] L. Bartolini, F. Bigazzi, S. Bolognesi, A. L. Cotrone, and A. Manenti, Theta dependence in holographic QCD, J. High Energy Phys. 02 (2017) 029.

[12] M. Järvinen and E. Kiritsis, Holographic models for QCD in the Veneziano limit, J. High Energy Phys. 03 (2012) 002. 
[13] D. Areán, I. Iatrakis, M. Järvinen, and E. Kiritsis, $C P$-odd sector and $\theta$ dynamics in holographic QCD, Phys. Rev. D 96, 026001 (2017).

[14] F. Brünner and A. Rebhan, Holographic QCD predictions for production and decay of pseudoscalar glueballs, Phys. Lett. B 770, 124 (2017).

[15] W. I. Eshraim, S. Janowski, F. Giacosa, and D. H. Rischke, Decay of the pseudoscalar glueball into scalar and pseudoscalar mesons, Phys. Rev. D 87, 054036 (2013).

[16] G. M. Shore and G. Veneziano, Renormalization group aspects of $\eta^{\prime} \rightarrow \gamma \gamma$, Nucl. Phys. B381, 3 (1992).

[17] P. Ball, J. M. Frère, and M. Tytgat, Phenomenological evidence for the gluon content of $\eta$ and $\eta^{\prime}$, Phys. Lett. B 365, 367 (1996).

[18] L. A. Harland-Lang, V. A. Khoze, M. G. Ryskin, and W. J. Stirling, Central exclusive production as a probe of the gluonic component of the $\eta^{\prime}$ and $\eta$ mesons, Eur. Phys. J. C 73, 2429 (2013).

[19] S. D. Bass and P. Moskal, $\eta^{\prime}$ and $\eta$ mesons with connection to anomalous glue, Rev. Mod. Phys. 91, 015003 (2019).

[20] C. Rosenzweig, A. Salomone, and J. Schechter, Pseudoscalar glueball, the axial anomaly and the mixing problem for pseudoscalar mesons, Phys. Rev. D 24, 2545 (1981).

[21] C. Rosenzweig, A. Salomone, and J. Schechter, How does a pseudoscalar glueball come unglued?, Nucl. Phys. B206, 12 (1982).

[22] R. Escribano, $J / \psi \rightarrow V P$ decays and the quark and gluon content of the $\eta$ and $\eta^{\prime}$, Eur. Phys. J. C 65, 467 (2010).

[23] H.-Y. Cheng, H.-n. Li, and K.-F. Liu, Pseudoscalar glueball mass from $\eta-\eta^{\prime}-G$ mixing, Phys. Rev. D 79, 014024 (2009).

[24] T. Gutsche, V. E. Lyubovitskij, and M. C. Tichy, $\eta(1405)$ in a chiral approach based on mixing of the pseudoscalar glueball with the first radial excitations of $\eta$ and $\eta^{\prime}$, Phys. Rev. D 80, 014014 (2009).

[25] V. Mathieu and V. Vento, Pseudoscalar glueball and $\eta-\eta^{\prime}$ mixing, Phys. Rev. D 81, 034004 (2010).

[26] KLOE collaboration, A global fit to determine the pseudoscalar mixing angle and the gluonium content of the $\eta^{\prime}$ meson, J. High Energy Phys. 07 (2009) 105.

[27] H.-W. Ke, X.-H. Yuan, and X.-Q. Li, Fraction of the gluonium component in $\eta^{\prime}$ and $\eta$, Int. J. Mod. Phys. A 26, 4731 (2011).

[28] C. J. Morningstar and M. J. Peardon, Glueball spectrum from an anisotropic lattice study, Phys. Rev. D 60, 034509 (1999).

[29] Y. Chen, A. Alexandru, S. Dong, T. Draper, I. Horvath et al., Glueball spectrum and matrix elements on anisotropic lattices, Phys. Rev. D 73, 014516 (2006).

[30] F. Brünner and A. Rebhan, Nonchiral Enhancement of Scalar Glueball Decay in the Witten-Sakai-Sugimoto Model, Phys. Rev. Lett. 115, 131601 (2015).

[31] F. Brünner and A. Rebhan, Constraints on the $\eta \eta^{\prime}$ decay rate of a scalar glueball from gauge/gravity duality, Phys. Rev. D 92, 121902 (2015).

[32] F. Brünner, D. Parganlija, and A. Rebhan, Glueball decay rates in the Witten-Sakai-Sugimoto model, Phys. Rev. D 91, 106002 (2015).
[33] UKQCD Collaboration, Glueball mass measurements from improved staggered fermion simulations, Phys. Rev. D 82, 034501 (2010).

[34] E. Gregory, A. Irving, B. Lucini, C. McNeile, A. Rago, C. Richards, and E. Rinaldi, Towards the glueball spectrum from unquenched lattice QCD, J. High Energy Phys. 10 (2012) 170.

[35] W. Sun, L.-C. Gui, Y. Chen, M. Gong, C. Liu, Y.-B. Liu, Z. Liu, J.-P. Ma, and J.-B. Zhang, Glueball spectrum from $N_{f}=2$ lattice QCD study on anisotropic lattices, Chin. Phys. C 42, 093103 (2018).

[36] D. V. Bugg, Data on $J / \Psi \rightarrow \gamma\left(K^{ \pm} K_{S}^{0} \pi^{\mp}\right)$ and $\gamma\left(\eta \pi^{+} \pi^{-}\right)$, arXiv:0907.3015.

[37] A. Masoni, C. Cicalo, and G. L. Usai, The case of the pseudoscalar glueball, J. Phys. G 32, R293 (2006).

[38] K. Becker, M. Becker, and J. Schwarz, String Theory and M-Theory: A Modern Introduction (Cambridge University Press, Cambridge, England, 2007).

[39] M. Kruczenski, D. Mateos, R. C. Myers, and D. J. Winters, Towards a holographic dual of large $N_{c}$ QCD, J. High Energy Phys. 05 (2004) 041.

[40] F. Bigazzi, A. L. Cotrone, and R. Sisca, Notes on Theta dependence in holographic Yang-Mills, J. High Energy Phys. 08 (2015) 090.

[41] R. C. Brower, S. D. Mathur, and C.-I. Tan, Glueball spectrum for QCD from AdS supergravity duality, Nucl. Phys. B587, 249 (2000).

[42] F. Brünner, J. Leutgeb, and A. Rebhan, A broad pseudovector glueball from holographic QCD, Phys. Lett. B 788, 431 (2019).

[43] G. S. Bali, F. Bursa, L. Castagnini, S. Collins, L. Del Debbio, B. Lucini, and M. Panero, Mesons in large-N QCD, J. High Energy Phys. 06 (2013) 071.

[44] P. H. C. Lau and S. Sugimoto, Chern-Simons five-form and holographic baryons, Phys. Rev. D 95, 126007 (2017).

[45] O. Bergman, S. Seki, and J. Sonnenschein, Quark mass and condensate in HQCD, J. High Energy Phys. 12 (2007) 037.

[46] A. Dhar and P. Nag, Tachyon condensation and quark mass in modified Sakai-Sugimoto model, Phys. Rev. D 78, 066021 (2008).

[47] O. Aharony and D. Kutasov, Holographic duals of long open strings, Phys. Rev. D 78, 026005 (2008).

[48] K. Hashimoto, T. Hirayama, F.-L. Lin, and H.-U. Yee, Quark mass deformation of holographic massless QCD, J. High Energy Phys. 07 (2008) 089.

[49] R. McNees, R. C. Myers, and A. Sinha, On quark masses in holographic QCD, J. High Energy Phys. 11 (2008) 056.

[50] V. Niarchos, Hairpin-Branes and Tachyon-Paperclips in holographic backgrounds, Nucl. Phys. B841, 268 (2010).

[51] P. Horava and E. Witten, Eleven-dimensional supergravity on a manifold with boundary, Nucl. Phys. B475, 94 (1996).

[52] G. J. Gounaris and H. Neufeld, Why $\imath$ (1460) decays mainly into $K \bar{K} \pi$ ?, Phys. Lett. B 213, 541 (1988).

[53] M. Tanabashi et al., Review of particle physics, Phys. Rev. D 98, 030001 (2018).

[54] N. Beisert and B. Borasoy, The $\eta^{\prime} \rightarrow \eta \pi \pi$ decay in $\mathrm{U}(3)$ chiral perturbation theory, Nucl. Phys. A705, 433 (2002).

[55] R. Escribano, P. Masjuan, and J. J. Sanz-Cillero, Chiral dynamics predictions for $\eta^{\prime} \rightarrow \eta \pi \pi$, J. High Energy Phys. 05 (2011) 094. 
[56] D. K. Hong, M. Rho, H.-U. Yee, and P. Yi, Chiral dynamics of baryons from string theory, Phys. Rev. D 76, 061901 (2007).

[57] H. Hata, T. Sakai, S. Sugimoto, and S. Yamato, Baryons from instantons in holographic QCD, Prog. Theor. Phys. 117, 1157 (2007).

[58] M. Rozali, J. B. Stang, and M. van Raamsdonk, Holographic Baryons from Oblate instantons, J. High Energy Phys. 02 (2014) 044.

[59] S. Bolognesi and P. Sutcliffe, The Sakai-Sugimoto soliton, J. High Energy Phys. 01 (2014) 078.

[60] J. Leutgeb, J. Mager, and A. Rebhan, Pseudoscalar transition form factors and the hadronic light-by-light contribution to the anomalous magnetic moment of the muon from holographic QCD, Phys. Rev. D 100, 094038 (2019).
[61] N. Anderson, S. K. Domokos, J. A. Harvey, and N. Mann, Central production of $\eta$ and $\eta^{\prime}$ via double Pomeron exchange in the Sakai-Sugimoto model, Phys. Rev. D 90, 086010 (2014).

[62] N. Anderson, S. Domokos, and N. Mann, Central production of $\eta$ via double Pomeron exchange and double Reggeon exchange in the Sakai-Sugimoto model, Phys. Rev. D 96, 046002 (2017).

[63] Z. Hu, B. Maddock, and N. Mann, A second look at stringinspired models for proton-proton scattering via Pomeron exchange, J. High Energy Phys. 08 (2018) 093.

[64] P. Lebiedowicz, O. Nachtmann, and A. Szczurek, Exclusive central diffractive production of scalar and pseudoscalar mesons; tensorial vs. vectorial pomeron, Ann. Phys. (Amsterdam) 344, 301 (2014). 